\title{
Traduire
}

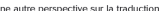

Revue française de la traduction

$244 \mid 2021$

Des jeux et des mots

\section{Traduire la criminalité. Perspectives traductologiques et discursives}

Sous la direction de Giuditta Caliendo et de Corinne Oster

Carine Bouillery

\section{CpenEdition}

\section{Journals}

Édition électronique

URL : https://journals.openedition.org/traduire/2370

DOI : $10.4000 /$ traduire.2370

ISSN : 2272-9992

Éditeur

Société française des traducteurs

Édition imprimée

Date de publication : 15 juin 2021

Pagination : 107-110

ISSN : 0395-773X

Référence électronique

Carine Bouillery, "Traduire la criminalité. Perspectives traductologiques et discursives », Traduire [En

ligne], 244 | 2021, mis en ligne le 15 juin 2021, consulté le 01 juillet 2021. URL : http://

journals.openedition.org/traduire/2370; DOI : https://doi.org/10.4000/traduire.2370 


\section{Lu pour vous}

Traduire la criminalité. Perspectives

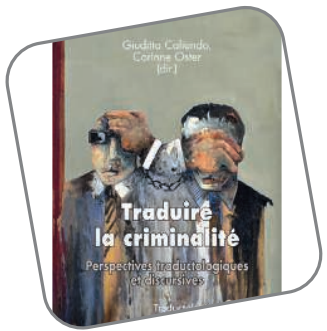
traductologiques et discursives Sous la direction de Giuditta Caliendo et de Corinne Oster

\section{Carine Bouillery}

Le crime est partout, même en traduction!

Les mafias opèrent dans un contexte international: elles n'hésitent pas à s'affranchir des frontières pour conduire leurs affaires. La réalité de ces groupes échappe souvent au grand public. Ainsi, la traduction devient un instrument fondamental pour la diffusion de l'information et le décryptage de ce phénomène.

Traduire la criminalité. Perspectives traductologiques et discursives se divise en quatre parties se concentrant chacune sur une forme de discours précise (littéraire, multimodal, cinématographique, juridique). Sous la direction de Giuditta Caliendo et Corinne Oster, les auteurs et autrices proposent de réfléchir sur les caractéristiques linguistiques du crime organisé et mettent au jour les difficultés rencontrées dans la traduction de notions complexes.

De simples termes peuvent être porteurs d'une idéologie si forte qu'ils doivent être choisis avec soin. Dans l'article consacré à la version anglaise du livre Mon traître de Sorj Chalandon, la traductrice Fiona McCann revient sur les difficultés de trouver les «bons mots» pour désigner les protagonistes du conflit entre l'IRA et l'armée britannique qui déchira pendant plus de trente ans l'Irlande du Nord. Dans un contexte aussi délicat, chaque parole sera perçue différemment selon la sensibilité du lecteur ou même sa situation géographique. 
Parfois, l'absence de termes adaptés dans la langue cible empêche de dépeindre une réalité intrinsèque à une culture source. Par exemple, la langue flamande ne possède pas de référence linguistique propre pour décrire une situation typiquement italienne. C'est pourquoi quand, lors de son traitement de l'affaire "Mafia Capitale" ${ }^{1}$ dans le quotidien flamand De Standaard, la journaliste Ine Roox qualifie l'organisation criminelle de "bande», elle masque totalement la dangerosité et le caractère mafieux du phénomène.

La guerre entre les cartels mexicains pour le contrôle du trafic de drogue a fait plus de 230000 morts entre 2006 et 2018, pourtant les groupes criminels sud-américains sont quasiment absents du panorama éditorial français. C'est ce trou que l'autrice Cathy Fourez tente d'expliquer dans l'ouvrage. Elle constate aussi que nombre des œuvres publiées en France sur le narcotrafic sont le fait d'auteurs états-uniens, laissant place à une interprétation filtrée de la réalité. Enfin, elle note que le Mexique présenté dans les traductions françaises est souvent un Mexique stéréotypé; elle émet l'hypothèse d'une politique de promotion gouvernementale visant à redorer (ou du moins à ne pas écorner) l'image du pays.

Au-delà de la transposition linguistique, la traduction est également un transfert interculturel délicat, et l'adaptation audiovisuelle n'échappe pas à la règle.

Dans son article, Giuseppe Balirano décortique les soustitres anglais des saisons 1 et 2 de la série Gomorra et démontre comment ils peuvent conduire à une perception erronée ou stéréotypée. Les dimensions proxémique (rapport à l'espace) et haptique (relative au toucher) diffèrent totalement au Royaume-Uni et en Italie. Ainsi, Ia relation hiérarchique entre deux hommes, où le «boss» affirme son pouvoir par une communication non verbale, a amené le public anglais à faire une lecture erronée des rapports physiques entre membres de la camorra. L'auteur note l'importance d'inclure dans les sous-titres des indications linguistiques claires et explicites qui accompagnent la compréhension de l'œuvre.

1. Scandale politico-financier qui a révélé l'infiltration criminelle de l'administration romaine. 
En analysant les sous-titres et la version doublée de deux films réalisés par Woody Allen sur la pègre italo-américaine, Frédérique Brisset relève quant à elle deux traitements différents. Elle constate que le doublage préfère garder les xénismes (terme emprunté tel quel à une langue étrangère, sans être traduit) pour marquer l'appartenance socioethnique du groupe criminel. La version sous-titrée, sans doute par manque de place, préfère les occulter, laissant le spectateur «orphelin» d'un élément essentiel à la caractérisation des personnages. L'autrice émet l'hypothèse que l'accès à la bande-son et la proximité étymologique entre le français et l'italien suffiront aux spectateurs pour comprendre le contexte.

Les problèmes globaux nécessitent une réponse globale.

\section{Don LUIGI CIOTTI}

Si la lutte contre la criminalité organisée passe par une sensibilisation accrue du public, elle se joue également sur le terrain judiciaire. La mafia a longtemps été considérée comme un phénomène typiquement italien, qui ne concernait pas les autres pays. On constate ces dernières années une réelle volonté européenne de combattre de front les groupes criminels.

Les deux derniers articles, consacrés à la traduction juridique, se penchent sur le manque d'harmonisation des textes législatifs et les difficultés terminologiques.

Le système italien étant le mieux «armé», la réponse judiciaire européenne s'est basée sur l'article 416bis du Code pénal transalpin introduisant l'associazione di tipo mafioso. La traduction de ce terme a rencontré plusieurs obstacles, notamment la présence de culturèmes: l'intimidazione (pouvoir intimidateur de l'association) et l'omertà (loi du silence) sont deux notions ancrées dans le territoire italien, difficilement transposables dans une autre culture. La transcription de l'article ne pouvait se contenter d'un simple calque de la langue source qui viderait le terme de sa substance: en effet, le mot association n'évoque aucune notion juridique

2. Prêtre italien, fondateur de «Libera», l'une des plus importantes associations italiennes antimafia. 
précise et n'exprime pas l'idée d'un groupe structuré comme la mafia. En revanche, la dernière définition en date montre de réels progrès: le concept proposé de "délit de participation à une organisation criminelle à caractère mafieux» est plus proche du reato mafioso où la seule appartenance à une organisation criminelle est condamnable.

La traduction juridique se heurte quant à elle à deux difficultés particulières. D'abord, la polysémie des termes légaux: au sein d'un même système, ils peuvent revêtir différentes acceptions selon le sous-domaine. Ensuite, les discordances existantes entre les codes nationaux (par exemple, la France compte trois infractions pénales, alors que les Pays-Bas n'en ont que deux). Les mafias étant des groupes internationaux, les infractions peuvent se produire dans un cadre transfrontalier: le traducteur doit non seulement être un linguiste hors pair, mais également un fin connaisseur des différents codes pénaux.

La volonté déclarée de l'ouvrage est de "remédier au peu d'attention qui a été accordé au rôle que jove la traduction dans les représentations discursives de la criminalité»: mission accomplie pour tous ces auteurs qui prouvent que la traduction est multiple, complexe, mais ô combien nécessaire. Un simple compte-rendu ne pourrait relater toute la richesse de cette œuvre collective.

CALIENDO Giuditta et OSTER Corinne (dir.) et al., Traduire la criminalité. Perspectives traductologiques et discursives, Villeneuved'Ascq, France, Presses universitaires du Septentrion, 2020. 